1998

\title{
Magnetic Properties OfCo/Rh (001) Multilayers Studied By X-Ray Magnetic-Circular Dichroism
}

\author{
M. A. Tomaz \\ E. Mayo \\ D. Lederman \\ E. Hallin \\ T. K. Sham \\ See next page for additional authors
}

Follow this and additional works at: https://researchrepository.wvu.edu/faculty_publications

\section{Digital Commons Citation}

Tomaz, M. A.; Mayo, E.; Lederman, D.; Hallin, E.; Sham, T. K.; O’Brien, W. L.; and Harp, G. R., "Magnetic Properties Of Co/Rh (001) Multilayers Studied By X-Ray Magnetic-Circular Dichroism" (1998). Faculty Scholarship. 587.

https://researchrepository.wvu.edu/faculty_publications/587 
Authors

M. A. Tomaz, E. Mayo, D. Lederman, E. Hallin, T. K. Sham, W. L. O’Brien, and G. R. Harp 


\title{
Magnetic properties of $\mathrm{Co} / \mathrm{Rh}(001)$ multilayers studied by $\mathrm{x}$-ray magnetic-circular dichroism
}

\author{
M. A. Tomaz \\ Department of Physics and Astronomy, Ohio University, Athens, Ohio 45701 \\ E. Mayo and D. Lederman \\ West Virginia University, Morgantown, West Virginia 26506-6315 \\ E. Hallin \\ Canadian Synchrotron Radiation Facility, University of Wisconsin-Madison, 3731 Schneider Drive, Stoughton, Wisconsin 53589 \\ T. K. Sham \\ Department of Chemistry, University of Western Ontario, London, Ontario, Canada NA6 5B7 \\ W. L. O’Brien \\ Synchrotron Radiation Center, University of Wisconsin-Madison, 3731 Schneider Drive, Stoughton, Wisconsin 53589 \\ G. R. Harp \\ Department of Physics and Astronomy, Ohio University, Athens, Ohio 45701
}

(Received 11 December 1997)

\begin{abstract}
The layer-averaged magnetic moments of $\mathrm{Co}$ and $\mathrm{Rh}$ have been measured in sputter deposited $\mathrm{Co} / \mathrm{Rh}(001)$ multilayer thin films using the x-ray magnetic circular dichroism. The Rh moments were measured at both the $L$ and $M$ absorption edges, where we find that the $\mathrm{Rh}$ moment decreases as a function of increasing $\mathrm{Rh}$ layer thickness $\left(t_{\mathrm{Rh}}\right)$. The decline of the layer-averaged $\mathrm{Rh}$ moment is well described in terms of a simple dilution, implying that the $\mathrm{Rh}$ moment is confined to the interfacial region. We find that the Co moment remains largely unaffected, maintaining a bulklike value of $1.7 \mu_{B}$ in the region preceding the first antiferromagnetic coupling peak where $t_{\mathrm{Rh}}$ ranges from 0 to $4 \AA$. We also find, via application of the dichroism sum rules, that the ratio $\left\langle L_{z}\right\rangle /\left\langle S_{z}\right\rangle$ for Co increases $\approx 10 \%$ for this same region. Finally, we contrast the magnetic behavior of the $\mathrm{Co} / \mathrm{Rh}(001)$ and $\mathrm{Fe} / \mathrm{Rh}(001)$ multilayer systems. [S0163-1829(98)07641-3]
\end{abstract}

\section{INTRODUCTION}

Magnetic multilayers comprised of bilayers of a ferromagnetic and a nonferromagnetic metal continue to command significant research efforts. The possibility of novel magnetic behavior, greater fundamental understanding, and applications potential equally fuel this work. The $\mathrm{Co} / \mathrm{Rh}$ system is one which exhibits oscillatory interlayer exchange coupling, ${ }^{1}$ and, thus, an enhanced magnetoresistance, which has been another motivation behind recent work. A review of recent work in terms of element-specific magnetic information finds that Rampe and co-workers have studied layers of $\mathrm{Rh}$ deposited on $\mathrm{Co}$ as well as $\mathrm{Co} / \mathrm{Rh} / \mathrm{Co}$ (0001) trilayers by spin- and angle-resolved photoemission ${ }^{2}$ and found that $\mathrm{Rh}$ showed a spin-polarized electronic structure (that is, a net magnetic moment). This spin polarization was significant at 1 atomic layer coverage but immeasurable at 3 atomic layers, quite similar to what Kachel et al. found for Rh on Fe (001). ${ }^{3}$ More recently, Zoll and co-workers ${ }^{4}$ studied Co/Rh (111) sandwiches and inferred from superconducting quantum interference device (SQUID) magnetometry that the Co therein retained its bulk magnetization. Furthermore, several calculations of $\mathrm{Co} / \mathrm{Rh}$ multilayers have indicated that Co retains its bulk moment while $\mathrm{Rh}$ is polarized at the interface whether in perfect multilayers or in the case of considerable intermixing. 4
Our motivation to study the crystallography and elementspecific magnetic moments of $\mathrm{Co} / \mathrm{Rh}$ (001) multilayers was partly based on our previous interesting results with $\mathrm{Fe} / \mathrm{Rh}$ (001) multilayers. ${ }^{7,8}$ Briefly, we found in the $\mathrm{Fe} / \mathrm{Rh}$ (001) system that the film underwent a body-centered tetragonal (bct) to face-centered tetragonal (fct) structural phase transition. Furthermore, Rh was ferromagnetic where the film had a bct structure and not so when the film morphed to the fct phase. The Fe moment was considerably enhanced in the bct phase and had no measurable moment in the fct phase. Thus, a better understanding of the role of the crystalline environment in $\mathrm{Rh}$ magnetic properties is desired. Our previous work has shown that $\mathrm{Co} / \mathrm{Rh}$ multilayers grow in a fct phase and so provides a way to study fct $\mathrm{Rh}$ in a different magnetic environment.

In this paper, we provide the results of element-specific magnetic moment measurements in $\mathrm{Co} / \mathrm{Rh}(001)$ multilayers by X-ray magnetic-circular dichroism (XMCD). ${ }^{9,10}$ Applying $\mathrm{XMCD}$ to the $\mathrm{Co} / \mathrm{Rh}$ system is nontrivial, yet CoRh alloys have been measured by this technique. ${ }^{11}$ The measurements in the multilayer system are more complicated than in alloys due to the antiferromagnetic (AF) coupling that the films exhibit over certain ranges of $t_{\mathrm{Rh}}$.

Additionally, XMCD of the $4 d$ elements is experimentally challenging. Recall that the $M$ edges (300-600 eV) are easily reached with conventional grating optics, yet have a 
much smaller absorption cross section than the $L$ edges. The $L$ edges $(2.5-3.5 \mathrm{keV})$ can be accessed with a crystal monochromator; however, the degree of circular polarization is a strongly varying function of photon energy for most crystals in this range. ${ }^{12}$ We employ both $\mathrm{Rh} L$ and $M$ edge spectra here where the $L$ edge spectra were obtained as part of a feasibility study. This work presents, to the best of our knowledge, the first quantitative, element-specific moment measurements of $\mathrm{Co} / \mathrm{Rh}(001)$.

\section{SAMPLE PREPARATION}

This paper presents data derived from the following samples. The first sample (S1) was deposited as $\left\{\mathrm{MgO}(001) / \mathrm{Cr} 4 \AA / \mathrm{Rh} 25 \AA /[\operatorname{Rh} 3 \AA / \mathrm{Co} 10 \AA]_{40} / \mathrm{Al} 20 \AA\right.$ \} The second sample (S2) was deposited as $\{\mathrm{MgO}(001) /$ Cr $4 \AA /$ Rh $25 \AA /[\text { Rh } 14 \AA / \text { Co } 10 \AA]_{20} / \mathrm{Al} 20 \AA$ \}. The third sample was prepared in the form of a wedge (W1) and deposited as $\left\{\mathrm{MgO}(001) / \mathrm{Cr} 4 \AA / \mathrm{Rh} 25 \AA /\left[\mathrm{Rh} t_{\mathrm{Rh}} / \mathrm{Co} 10 \AA\right]_{20} /\right.$ Al $20 \AA$ \}, where $t_{\mathrm{Rh}}$ is varied from 0 to $10 \AA$. The final sample was also prepared in the form of a wedge (W2) and deposited as $\left\{\mathrm{MgO}(001) / \mathrm{Cr} 4 \AA / \mathrm{Rh} 25 \AA /\left[\mathrm{Rh} t_{\mathrm{Rh}} / \mathrm{Co} 10\right.\right.$ $\AA]_{20} / \mathrm{Al} 20 \AA$, where $t_{\mathrm{Rh}}$ is varied from 0 to $50 \AA$. The single-thickness films S1 and S2 were deposited on $1 \mathrm{~cm} \times 1 \mathrm{~cm}$ square substrates. The wedge samples $\mathrm{W} 1$ and W2 were deposited on $50 \mathrm{~mm} \times 8 \mathrm{~mm}$ rectangular substrates where the wedge portion of the film was limited to $40 \mathrm{~mm}$. Rh gradients are $0.25 \AA / \mathrm{mm}$ and $1.25 \AA / \mathrm{mm}$ for $\mathrm{W} 1$ and $\mathrm{W} 2$, respectively.

All films used in the study were prepared by dc magnetron sputtering onto single-crystal $\mathrm{MgO}$ (001)-oriented substrates. Polished $\mathrm{MgO}$ (001) substrates used for the films described above were briefly repolished with $0.05 \mu \mathrm{m}$ alumina and $\mathrm{H}_{2} \mathrm{O}$, rinsed, and then introduced to the sputtering chamber via a load lock transfer mechanism. All substrates were outgassed for a minimum of $0.5 \mathrm{~h}$ at $\geqslant 550{ }^{\circ} \mathrm{C}$ prior to any material deposition. $\mathrm{Cr}$ seed and $\mathrm{Rh}$ base layers were subsequently deposited at $550{ }^{\circ} \mathrm{C}$ onto these $\mathrm{MgO}(001)$ substrates. The films were then allowed to cool in the ambient vacuum (approximately $2 \mathrm{~h}$ ) prior to deposition of the multilayer. The growth temperature for the multilayers was $200{ }^{\circ} \mathrm{C}$. Upon completion of the multilayer, a protective capping layer of $20 \AA$ aluminum was deposited at less than $100{ }^{\circ} \mathrm{C}$

The $4 \AA \mathrm{Cr}$ seed layer was used as it is has been shown to provide a useful transition layer for systems with large lattice mismatches. ${ }^{13}$ The mismatch of $\mathrm{Rh}$ on $\mathrm{MgO}$ represents a 9.7\% expansion of $\mathrm{Rh}$ in the plane whereas the mismatch of $\mathrm{Cr}$ on $\mathrm{MgO}$ represents only a 3.2\% expansion of the $\mathrm{Cr}$ in the plane. Thus the $\mathrm{Cr}$ serves as a lattice matching template for the $\mathrm{Rh}$ as the in-plane expansion of $\mathrm{Rh}$ on $\mathrm{Cr}$ is only $6.8 \%$. The multilayer components $\mathrm{Co}$ and $\mathrm{Rh}$ have a $7 \%$ mismatch between them. The calculated difference in the surface free energy, which is a measure of the tendency toward surface segregation, for $\mathrm{Co}$ and $\mathrm{Rh}$ at $200{ }^{\circ} \mathrm{C}$ is small at approximately 5.2\%. ${ }^{14}$ Samples S1 and S2 were prepared primarily for examining structure in this system while the wedges W1 and W2 were to study both structure and the magnetic behavior as a function of $t_{\mathrm{Rh}}$.



FIG. 1. High- and low-angle XRD $\theta-2 \theta$ scan for the S1 and S2 samples.

\section{STRUCTURAL AND MAGNETIC CHARACTERIZATION}

\section{A. Structural characterization by x-ray diffraction}

The films were characterized by high-angle specular x-ray diffraction (XRD) using a fixed anode diffractometer with $1^{\circ}$ angular resolution, two-circle goniometer, and $\mathrm{Cu} K \alpha$ radiation. It is useful to mention that the wedge sample was examined with slits to provide the most narrow beam in our instrument. The incident beam width varies from approximately $2 \mathrm{~mm}$ at $25^{\circ}$ to $1 \mathrm{~mm}$ at $50^{\circ}$. Thus the maximum thickness which is averaged over in the XRD data of W1 is of order $0.5 \AA$. This point is of no concern for samples S1 and $\mathrm{S} 2$.

The XRD results from S1 and S2 are shown in Fig. 1. The upper panel shows both low- and high-angle $\theta-2 \theta$ scans of $\mathrm{S} 1$. There is a single $\mathrm{Co} / \mathrm{Rh}(002)$ multilayer feature with a broad shoulder at the low-angle side which is due to the $\mathrm{Rh}$ buffer (002) reflection. The double-peak structure in the lowangle scan (inset) is a result of an accidental change in the Co deposition thickness from $10 \AA$ to $8.5 \AA$ in the course of deposition. The peak positions correspond to bilayer periods of $12.2 \AA$ and $13.8 \AA$ which is in very good agreement with the nominal values of 11.5 and $13 \AA$. The lower panel of Fig. 1 presents the high- and low-angle $\theta-2 \theta$ results from S2. The high-angle scan shows the principle $\mathrm{Co} / \mathrm{Rh}(002)$ reflection as well as $n= \pm 1,+2$ satellite peaks. The low-angle portion of the scan shows three superlattice (SL) peaks which confirms that the film is well modulated. Analysis of the SL and multilayer (ML) reflections yields a bilayer thickness of $25 \AA$ in good agreement with the nominal deposited value of $24 \AA$. The apparent plateau between the $n=0$ and $n=-1$ peaks is real and is associated with the $\mathrm{Rh}$ base layer. This $\mathrm{Rh}$ base layer peak is evident in all the XRD scans of W1.

Figure 2 shows the (001) average superlattice interplanar spacing for $\mathrm{W} 1$ deduced from the position of the main superlattice peak $(n=0)$. We see that for $t_{\mathrm{Rh}}=0$, the spacing is slightly less than that for bulk Co as expected from the mismatch of $\mathrm{Co}$ on the $\mathrm{Rh}$ base layer. The spacing then increases up to $t_{\mathrm{Rh}}=2-3 \AA$. The spacing falls to a plateau at 


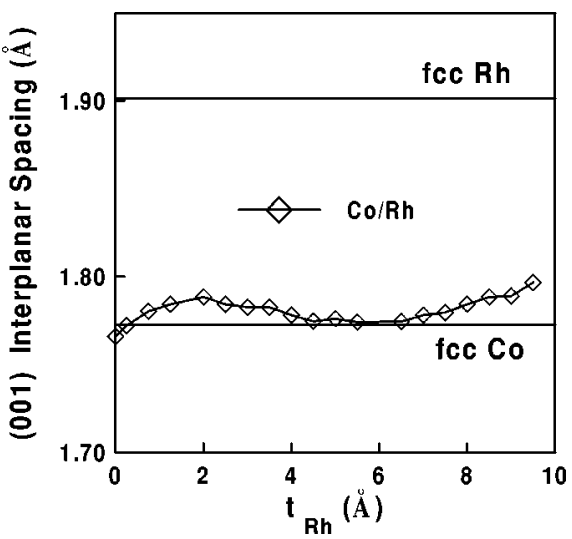

FIG. 2. The interplanar spacing in the (001) growth direction as a function of $t_{\mathrm{Rh}}$ for the sample W1.

$t_{\mathrm{Rh}}=4-6.5 \AA$ and then increases through $t_{\mathrm{Rh}}=10 \AA$. We propose that as $\mathrm{Rh}$ is added, the lattice mismatch stresses the film, resulting in an increased (001) interplanar spacing. Eventually, dislocations are introduced, allowing the film to relax in plane and reducing the perpendicular lattice spacing. Subsequently the spacing increases again as would be expected from the addition of material with a larger lattice constant.

In order to obtain information about the in-plane film crystallography, x-ray diffraction of the S1 and S2 superlattices was performed using a $18 \mathrm{~kW}$, rotating-anode $\mathrm{x}$-ray diffractometer equipped with a four-circle goniometer, using $\mathrm{Cu} K \alpha$ radiation. The in-plane lattice parameter was determined from Fig. 3 which shows $\theta-2 \theta$ scans of S1 and S2 with the wave vector $\mathbf{q}$ along the [113] direction, indexing on a cubic unit cell. From the position of the (113) peak, and using the out-of-plane lattice parameters of $c=3.60 \AA$ for S1 and $c=3.61 \AA$ for $\mathrm{S} 2$, the average in-plane lattice parameter of the superlattice was determined to be $a=3.81 \AA$ for S1 and $a=3.74 \AA$ for $\mathrm{S} 2$. This shows that the samples are facecentered tetragonal, with approximately $6 \%$ and $3 \%$ distortions for S1 and S2, respectively. The insets in each panel are rocking curves of the (113) reflections which possess full width at half maximum (FWHM) of less than $0.1^{\circ}$.

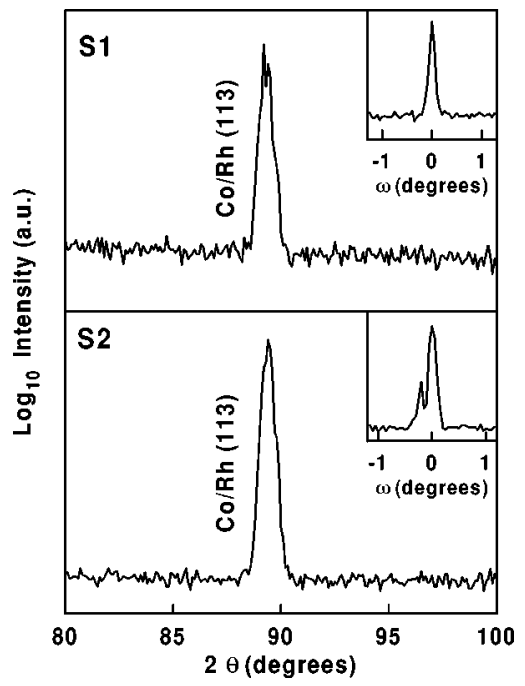

FIG. 3. The XRD $\theta-2 \theta$ scan for the $\mathrm{S} 1$ and $\mathrm{S} 2$ samples along the (113) crystallographic direction and rocking curves of the peaks.

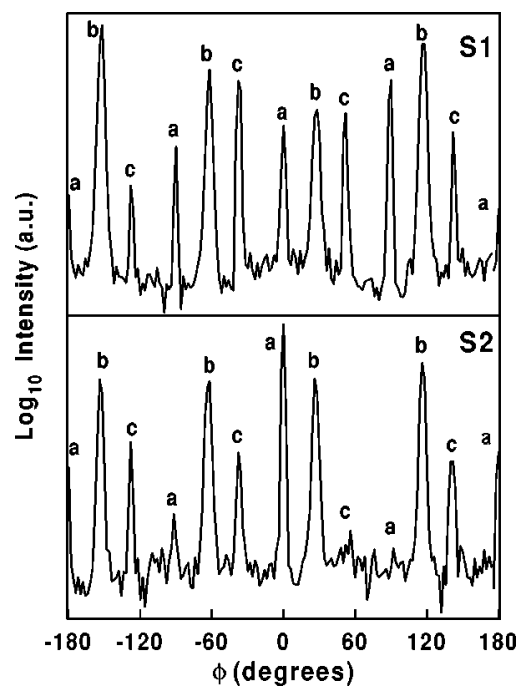

FIG. 4. The in-plane XRD $\phi$ scans for the S1 and S2 samples.

Figure 4 shows $\phi$ scans of the (113) reflection where the sample is rotated about the [001] direction, while $\mathbf{q}$ remains fixed at the superlattice (113) Bragg condition. The upper panel is for S1 while the lower is of S2. In both cases 12 peaks are visible, with a set of 3 peaks repeating every $90^{\circ}$, showing the expected fourfold symmetry. In each set the center peak (e.g., $\phi=-58$ ) corresponds to the film (113) peak, and is oriented with the [001] direction along the $\mathrm{MgO}$ [001]. The peaks to the left and the right of the center peak are $26^{\circ}$ away from the center peak and correspond to the sample's [001] direction parallel to the $\mathrm{MgO}$ [210] and [210] directions. The three peaks therefore correspond to three in-plane domains within the film. Clearly, the three in-plane domains are evident in S1 and S2 which indicates that the film morphology is similar regardless of $t_{\mathrm{Rh}}$ for this thickness.

In summary, we conclude that the films are well modulated and highly ordered in the (001) growth direction with a fct crystal structure. The growth is quasiepitaxial since there are three in-plane domains with well-defined in-plane crystallographic directions with respect to the $\mathrm{MgO}$ substrate. Interestingly, the perpendicular lattice constant remains within $3 \%$ of the bulk Co value for $t_{\mathrm{Rh}}$ up to $10 \AA$.

\section{B. Magneto-optic Kerr effect magnetometry}

Magneto-optical Kerr effect (MOKE) hysteresis loops were measured for all films on a Kerr effect magnetometer described elsewhere. ${ }^{7}$ The spot size of the laser in our Kerr effect instrument is approximately $1 \mathrm{~mm}$ in diameter. The $\mathrm{Rh}$ thickness averaged over is $0.25 \AA$ and $1.25 \AA$ for $\mathrm{W} 1$ and W2, respectively. Figure 5 shows a sequence of loops from $\mathrm{W} 1$ for various $t_{\mathrm{Rh}}$ as well as the hysteresis loop of a pure Co sample. The sequence shows the progression from ferromagnetic (FM) coupling through AF coupling and the return to FM coupling (though not completely FM coupled). Closer inspection of the sequence shows that the $\mathrm{Co}$ in the multilayer film is initially bulklike as the pure Co and $t_{\mathrm{Rh}}$ $=0 \AA$ loops are essentially identical. Moving to $t_{\mathrm{Rh}}=3 \AA$ we see a reduction in the maximum Kerr signal $\theta_{K}$ accompanied by an increase in the coercivity. This trend continues through 


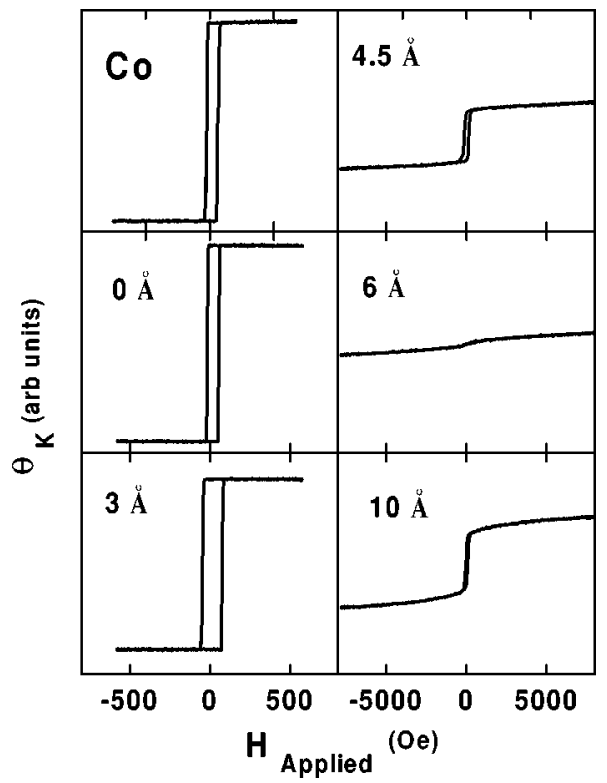

FIG. 5. Hysteresis loop of the pure Co sample followed by a sequence of MOKE loops taken from W1. The loops show the progression from FM to AF and back to FM coupling. The number in the upper left hand corner denotes $t_{\mathrm{Rh}}(\AA)$. Note that the applied field scale is 10 times greater in the right hand panels.

the loop for $t_{\mathrm{Rh}}=4.5 \AA$ along with a reduction in loop squareness. The film is clearly AF coupled by $t_{\mathrm{Rh}}=6 \AA$ and we estimate the peak of the $\mathrm{AF}$ coupling to occur at $t_{\mathrm{Rh}}$ $=7.2 \AA$. The film then begins to return to a FM-coupled state as seen in the loop for $t_{\mathrm{Rh}}=10 \AA$. Note that the $\theta_{K}$ scale is the same in each panel while the applied field scale is 10 times greater in the right hand panels. Additionally, the film does not saturate in an applied field of $8 \mathrm{kOe}$ in the range where $t_{\mathrm{Rh}}=5-9 \AA$.

Figure 6 summarizes the Kerr effect data from W2 (note that similar results were obtained on W1 though limited to a

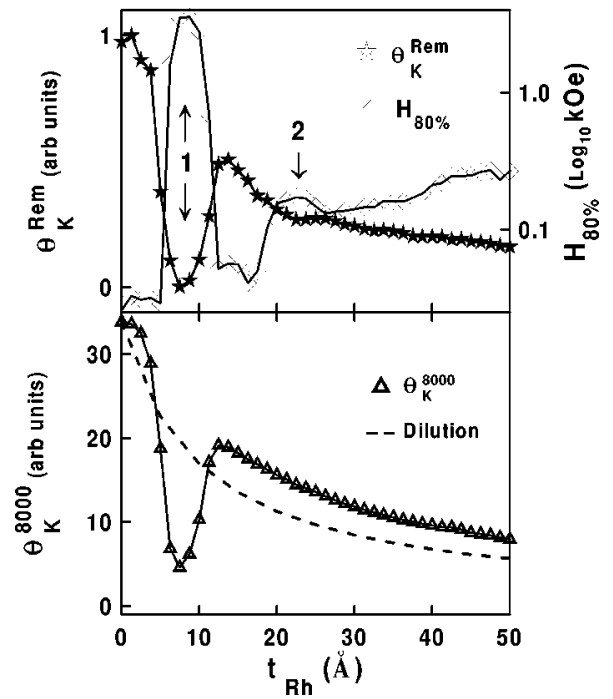

FIG. 6. Upper panel shows the remanant Kerr response and the $80 \%$ saturation field as functions of $t_{\mathrm{Rh}}$ for W2. Note the AF coupled region could not be saturated. The lower panel shows the Kerr response at an applied field of 8000 Oe and a dilution model plotted as functions of $t_{\mathrm{Rh}}$ for $\mathrm{W} 2$.
Rh thickness of $10 \AA$ ). The upper panel shows plots of $\theta_{K}^{\text {Rem }}$ which represents the Kerr response at zero applied field and $H_{80 \%}$ which is the value of applied field at which the sample achieves $80 \%$ of the maximum magnetization, as functions of $t_{\mathrm{Rh}}$. Both curves indicate the transition from FM to AF to FM coupling and two such transitions are apparent in W2. The second AF region of $\mathrm{W} 2$ is much weaker though clearly evident in both plots.

The lower panel of Fig. 6 shows a plot of $\theta_{K}^{8000}$ as a function of $t_{\mathrm{Rh}}$ where $\theta_{K}^{8000}$ represents the Kerr response at an applied field of 8000 Oe. Here again we see the coupling proceed through the FM to AF to FM sequence. The second AF region is not visible in this plot since the coupling weakens considerably there and the film is able to be saturated in the $8 \mathrm{kOe}$ field. An additional plot of a simple dilution is also shown which has the form

$$
\text { dilution }=\operatorname{MAX}\left(\theta_{K}^{8000}\right)\left(\frac{t_{\mathrm{Co}}}{t_{\mathrm{Co}}+t_{\mathrm{Rh}}}\right) \text {. }
$$

This model follows the outline of the Kerr effect quite well. We will discuss this further in the section on XMCD.

Finally, loops taken at different azimuthal angles yielded easy and hard magnetization axis orientations consistent with the underlying crystal structure. The (100) crystal planes have a fourfold symmetry, and thus four in-plane easy directions separated by $90^{\circ}$ were observed along the $\langle 110\rangle$ directions of the fct superlattice.

\section{X-ray magnetic-circular dichroism}

The XMCD signal being proportional to the magnetization ${ }^{15}$ implies that the largest signals can be obtained in the saturated state. However, the best data are obtained by measuring the samples in a remanant state as measurements made in an applied field suffer from a detection asymmetry between the two orientations of applied field which is due to the detection technique. Thus a high degree of remanence provides better data. In fact, the $\mathrm{Co} / \mathrm{Rh}$ system exhibits a strong interlayer oscillatory exchange coupling which makes moment measurements more difficult in regions with $\mathrm{AF}$ coupling and thus limits the ranges of measurement. All XMCD results presented below are based on absorption spectra obtained in the remanant state after application of a 1500 Oe applied field which was sufficient to saturate the film except for the range where $t_{\mathrm{Rh}}=5-9 \AA$ on sample W1.

Element-specific moments were determined by analyzing XMCD spectra obtained with a method described elsewhere. ${ }^{7}$ The reference samples used in the analysis were

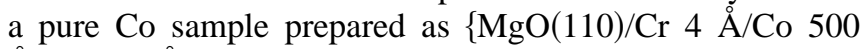
$\AA / A l \quad 20 \AA\}$ for the Co spectra and an alloy film of $\mathrm{Fe}_{0.75} \mathrm{Rh}_{0.25}$ prepared by codeposition was used as a standard for analysis of the Rh XMCD spectra. The use of reference spectra has previously been used to measure element specific moments in $\mathrm{Fe} / \mathrm{Cr}, \mathrm{Fe} / \mathrm{V}, \mathrm{Fe} / \mathrm{Rh}$, and $\mathrm{Fe} / \mathrm{Ru}$ multilayers via $\mathrm{XMCD}{ }^{7,8,16-18}$

In this paper we present both $L$ and $M$ edge results on $\mathrm{Rh}$, and though a detailed description of $L$ vs $M$ edge XMCD appears elsewhere, ${ }^{12}$ it is useful to briefly review it here. The $L$ edge absorption and dichroism involve the $2 p_{3 / 2}$ and $2 p_{1 / 2}$ 


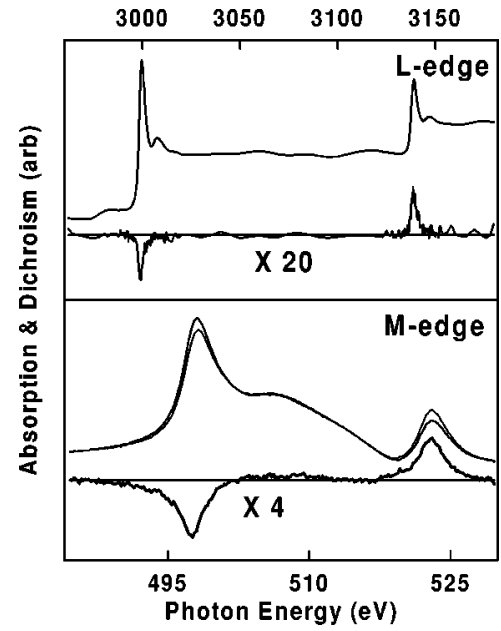

FIG. 7. A comparison of Rh $L$ and $M$ edge absorption and dichroism spectra. A large constant background has been subtracted from the $M$ edge spectrum to bring out its detail. Note the sharpness of the $L$ edge absorption features in comparison to the $M$ edge.

states whereas the $M$ edge involves the $3 p_{3 / 2}$ and $3 p_{1 / 2}$ states. The most obvious difference is the binding energies of the states involved. Figure 7 shows the absorption and dichroism spectra of $\mathrm{Rh}$ from an $\mathrm{Fe}_{0.75} \mathrm{Rh}_{0.25}$ alloy for both the $L$ and $M$ edges. The $M_{2}$ and $M_{3}$ edges are at 522 and 498 $\mathrm{eV}$, respectively; thus there is a $24 \mathrm{eV}$ spin-orbit (SO) splitting. The $L_{2}$ and $L_{3}$ edges are at 3140 and $3000 \mathrm{eV}$, respectively, representing a $140 \mathrm{eV}$ SO splitting. This larger splitting makes for more clearly defined peaks with no overlap. The $L$ edge absorption has a stronger signature above the background. Another important issue is the data collection time. The $L$ edge spectra shown were obtained in a single 2 h scan whereas the $M$ edge spectra shown represent some 30 scans taking $15 \mathrm{~h}$ in total. As stated previously, the $L$ edge spectra were obtained as part of a feasibility study and thus are incorporated here as complementary to the $M$ edge data.

The $L$ edge spectra were obtained on the Canadian Double Crystal Monochromator at the synchrotron radiation center in Stoughton, WI with InSb $\langle 111\rangle$ crystals. We have calculated an estimate of the degree of circular polarization and find it to vary from $38 \%$ to $53 \%$ in the $\mathrm{Rh} L$ edge energy range. ${ }^{12}$ Note that at higher energies, the degree of polarization is greater; thus the $L_{3}$ dichroism peak is suppressed relative to the $L_{2}$. There has been no attempt made to correct the spectra for this imbalance as the moment measurement method we use only requires that the spectra being compared be taken under the same conditions. ${ }^{7,16}$

The $\mathrm{Rh}$ moments as measured by XMCD are presented in Fig. 8. Both $L$ and $M$ edge measurements are presented and are in very good agreement with each other where measured at both edges (not shown). The Rh moment appears to initially increase or stay relatively constant up to $t_{\mathrm{Rh}}=3 \AA$ and then begins to decay away. The increase to an apparent maximum is probably real and can be understood from the standpoint of what can be expected to occur at the interface. One possibility is a surface polarization of $\mathrm{Rh}$; the other is an interdiffusion region of a couple of monolayers. Thus we anticipate a maximum moment in the neighborhood of the maximal interfacial region after which the layer average moment would decay.

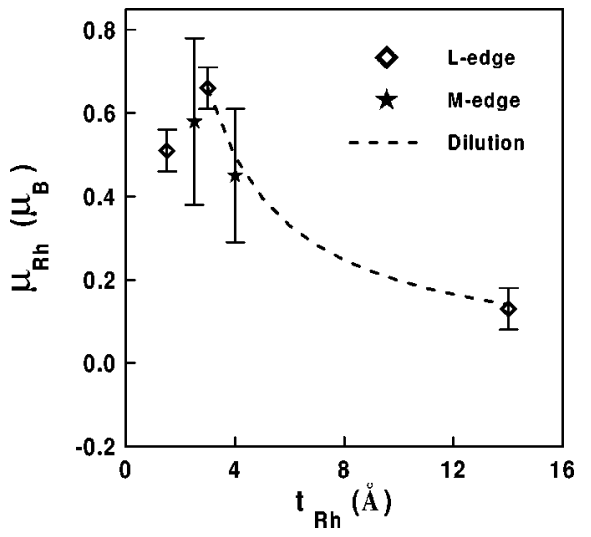

FIG. 8. The Rh moments as determined by XMCD. The point at $t_{\mathrm{Rh}}=14 \AA$ is corrected for finite remanence. The dashed line represents a dilution as described in the text.

The decay beyond $t_{\mathrm{Rh}}=3 \AA$ can be interpreted by a simple model where the moment on the $\mathrm{Rh}$ is assumed to exist only at the interface. Thus when more Rh is added, it simply dilutes the Rh XMCD. The observed decay is well fit by this simple dilution model, taking the maximum moment value as the starting point. Thus

$$
\mu_{\mathrm{Rh}}(t)=\mu_{\mathrm{Rh}}^{\max }\left(3 \AA / t_{\mathrm{Rh}}\right) \text {. }
$$

This is the dashed line curve plotted in Fig. 8. Note that no points where $t_{\mathrm{Rh}}=5-9 \AA$ are included as the film is $\mathrm{AF}$ coupled there. Additionally, the point at $t_{\mathrm{Rh}}=14 \AA$ has been corrected for finite remanence. This model is equivalent to that presented as Eq. (3) of Ref. 16 using a value of $\lambda=0$.

The layer-averaged $\mathrm{Rh}$ moment is aligned ferromagnetically to the Co moment as determined by the sign of the dichroism. This alignment of the $\mathrm{Rh}$ moment persists at all values of $t_{\mathrm{Rh}}$ and is similar to that observed in CoRh alloys. ${ }^{11}$ However, note that in bulk $\mathrm{Co}_{x} \mathrm{Rh}_{1-x}$ alloys, the Co moment decreases monotonically with increasing $\mathrm{Rh}$ concentration, to $1.34 \mu_{B}$ at $x=0.77$ and to $0.45 \mu_{B}$ at $x=0.49$. The fact that the Co moments do not decrease with increasing $t_{\mathrm{Rh}}$ in the present samples argues against strong interdiffusion between the $\mathrm{Co}$ and $\mathrm{Rh}$ layers. The $\mathrm{Rh}$ moments in the above mentioned alloys were $0.64 \mu_{B}$ and $0.11 \mu_{B}$, respectively. This suggests that the Rh moment decreases more rapidly than the Co moment, as each $\mathrm{Rh}$ atom acquires (on average) more $\mathrm{Rh}$ nearest neighbors. This is consistent with the present data, where Rh atoms away from the Co interface appear to have negligible moment.

The Co XMCD results are given in Fig. 9 where we see a trend similar to that observed in the $\theta_{K}^{\text {Rem }}$ data of Fig. 6 . There is a significant difference however; the Co moments do not fall off until $t_{\mathrm{Rh}}=3.5 \AA$ whereas the decline in $\theta_{K}^{\text {Rem }}$ begins immediately. The remanant Co moments only begin to decline as the Co layers begin to couple antiferromagnetically, whereas the decline in $\theta_{K}$ is a complex function of several factors, the primary one being a dilution of the overall magnetization with the addition of Rh. The primary result here is that to within the limits of the XMCD technique, the saturation Co moment is constant with increasing $t_{\mathrm{Rh}}$. This is in agreement with previous experimental ${ }^{4}$ and theoretical ${ }^{5,6}$ work. 


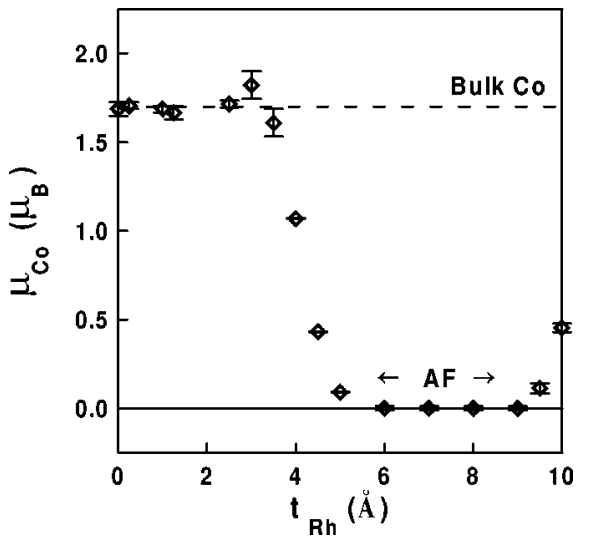

FIG. 9. The Co moments as determined by XMCD from W1. Note that the moment is bulklike until the film begins to couple antiferromagnetically.

We have applied the dichroism sum rules ${ }^{19}$ to the Co XMCD to determine the $L_{z} / S_{z}$ ratio. Figure 10 shows the absorption and dichroism spectra for the pure Co film along with the integrals representing $L_{z}$ and $S_{z}$ where the end points as noted in the figure give the relevant values. It should be noted that the integral denoting $S_{z}$ neglects the dipole correction term $\frac{7}{2} T_{z}$ which should be a good approximation [a first principles band calculation for hcp Co yields a $T_{z} / S_{z}$ ratio of only $-0.26 \%$ (Ref. 20)]. Evaluation of the integrals yields an $L_{z} / S_{z}$ ratio of 0.197 where gyromagnetic ratio measurements ${ }^{2 i}$ yield 0.194 for a most excellent agreement. A previous XMCD measurement on pure Co yielded a value of $0.196,{ }^{22}$ also in excellent agreement.

Figure 11 shows the $L_{z} / S_{z}$ ratio as a function of $t_{\mathrm{Rh}}$ in the range of $0-4 \AA$. The increase in the ratio of $L_{z} / S_{z}$ is not unexpected ${ }^{23}$ as the mere existence of an interface may provide the physical explanation. It is interesting, however, that the ratio of $L_{z} / S_{z}$ increases while the total Co moment remains constant. A potential explanation lies in the band hybridization between Co and Rh. Recall that these two elements are isoelectronic and have atomic ground state electronic configurations of $3 d^{8} 4 s^{1}$ for $\mathrm{Co}$, while $\mathrm{Rh}$ is $4 d^{8} 5 s^{1}$. Any $d-d$ hybridization could increase the effective spin-orbit splitting of the Co orbitals and thus increase the orbital moment. The fact that the effect appears to saturate is consistent with this notion as one only expects significant hybridization at the interface.

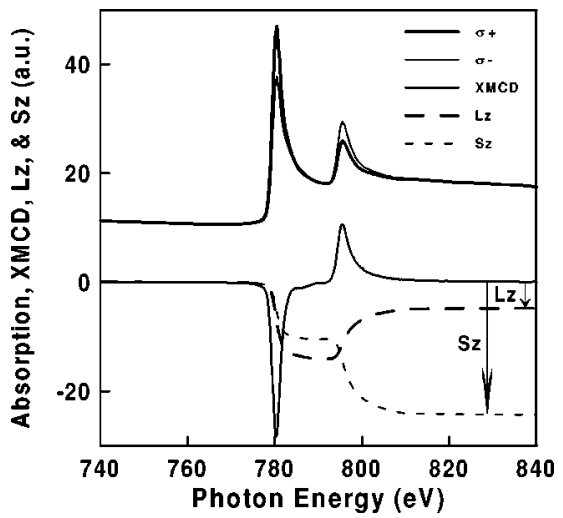

FIG. 10. The Co absorption and dichroism spectra from the pure Co sample. The $L_{Z}$ and $S_{Z}$ integrals are also shown.

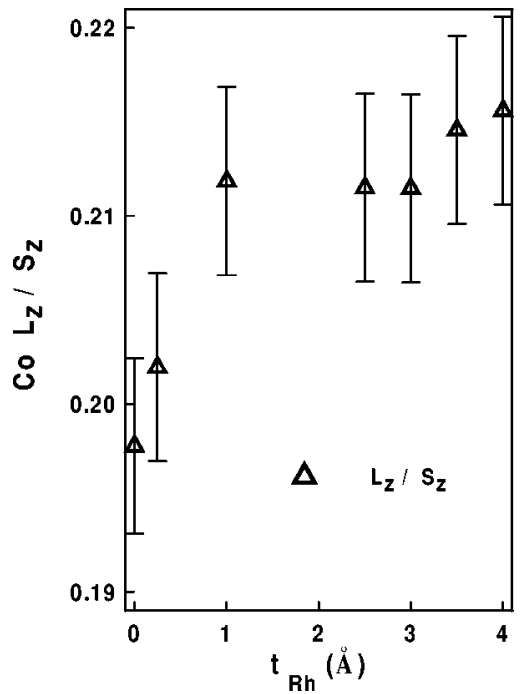

FIG. 11. The $L_{Z} / S_{Z}$ ratio in the Co moments of W1 as a function of $t_{\mathrm{Rh}}$.

\section{COMPARISON TO Fe/Rh (001) MULTILAYERS}

A comparison of $\mathrm{Fe} / \mathrm{Rh}(001)$ and $\mathrm{Co} / \mathrm{Rh}(001)$ multilayers reveals the relationship between the crystalline environment and magnetic environment on the induced $\mathrm{Rh}$ magnetism. Figure 12 shows the (001) interplanar spacing for the two systems as derived from XRD. In both cases, the ferromagnetic layer is $10 \AA$ while the Rh layer was deposited in the form of a wedge. The $\mathrm{Fe} / \mathrm{Rh}$ system shows a strong dependence on Rh thickness. In the bct region $\left(t_{\mathrm{Rh}} \leqslant 5 \AA\right)$ as well as in the fcc region $\left(t_{\mathrm{Rh}} \geqslant 5 \AA\right)$, a linear increase in the interplanar spacing is observed. It appears that the structure of $\mathrm{Rh}$ dominates the structure in the $\mathrm{Fe} / \mathrm{Rh}$ system. The $\mathrm{Co} / \mathrm{Rh}$ structure is essentially unchanged over this range of $t_{\mathrm{Rh}}$ with an interplanar spacing close to that of pure Co. Thus it appears here that Co dominates the structure in the $\mathrm{Co} / \mathrm{Rh}$ system.

The measured magnetic moments are presented in Fig. 13. The lower panel presents the $\mathrm{Fe}$ and $\mathrm{Co}$ moments. It is clear that the Fe is enhanced above its bulk value for $t_{\mathrm{Rh}} \leqslant 5 \AA$ (the bct region) and then drops off. The Co moments, on the other

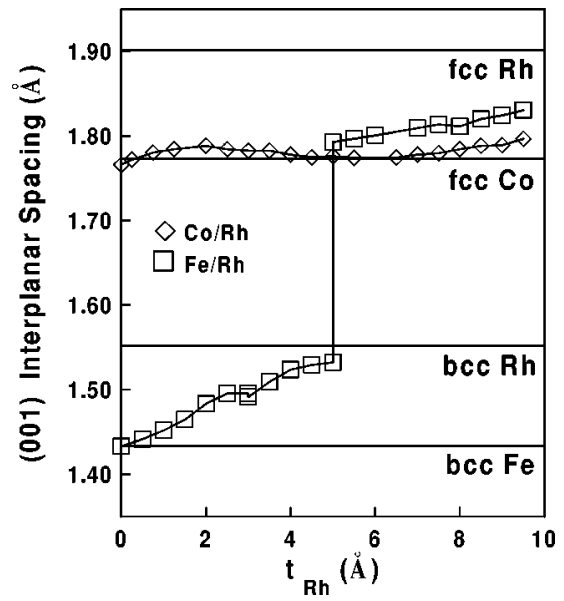

FIG. 12. A comparison of the (001) interplanar spacing for similar $\mathrm{Co} / \mathrm{Rh}$ and $\mathrm{Fe} / \mathrm{Rh}$ multilayer wedge samples. The FM layer is 10 $\AA$ while $t_{\mathrm{Rh}}$ varies from 0 to $10 \AA$. 


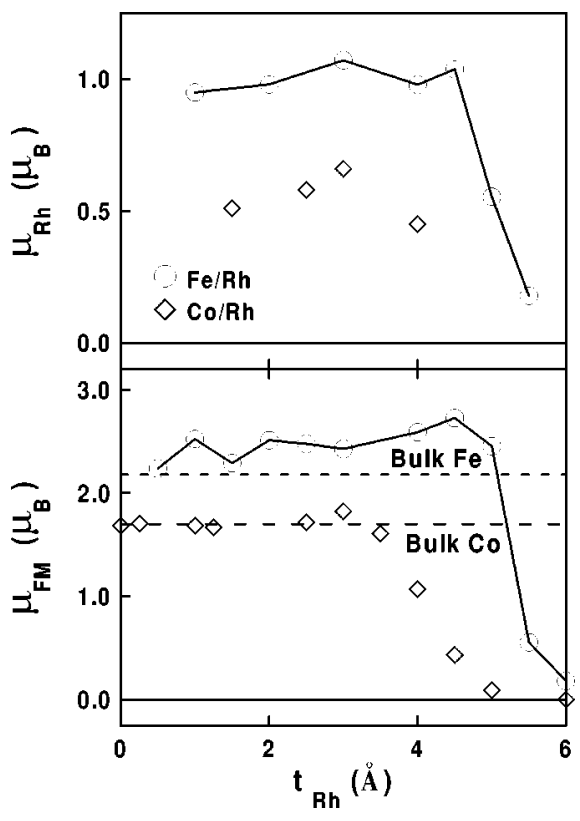

FIG. 13. A comparison of the FM and Rh moments from similarly prepared $\mathrm{Co} / \mathrm{Rh}$ and $\mathrm{Fe} / \mathrm{Rh}$ multilayer wedge samples.

hand, are constant prior to their decline. Although both $\mathrm{Fe}$ and Co show sharp reductions in moment, the reasons are very different. In the case of $\mathrm{Fe}$, the structure goes fct and the Fe becomes paramagnetic. In the Co case, the reduction is due to the onset of AF coupling. We wish to emphasize that the Fe/Rh (001) system cannot show this coupling since the $\mathrm{Fe}$ is no longer magnetic.

The upper panel of Fig. 13 shows the Rh moments in the two systems. The $\mathrm{Rh}$ in the bct $\mathrm{Fe} / \mathrm{Rh}$ multilayer exhibits a large moment which is on average $1 \mu_{B}$. In the fct region of the $\mathrm{Fe} / \mathrm{Rh}$ film, the $\mathrm{Rh}$ becomes paramagnetic similarly to $\mathrm{Fe}$. The $\mathrm{Rh}$ moment in the $\mathrm{Co} / \mathrm{Rh}$ films is significantly smaller at an average of $0.6 \mu_{B}$ in the region prior to the onset of AF coupling. In the $\mathrm{Co} / \mathrm{Rh}$ case, the decline in the layer-averaged $\mathrm{Rh}$ moment is due to the fact that only the interfacial $\mathrm{Rh}$ atoms are polarized and the additional $\mathrm{Rh}$ atoms are not.

Thus we conclude that a $\mathrm{Rh}$ moment is supported by proximity to a ferromagnet while the magnitude of the moment is related to the crystalline environment. The magnitude of the Rh moment may in part be related to the density of the bcc vs fcc structure. It has been shown that expansion of the $\mathrm{Rh}$ lattice will eventually yield a magnetic ground state. ${ }^{24}$ If we compare the ratio of $\mu_{\mathrm{Rh}} / \mu_{\mathrm{FM}}$ for $\mathrm{Fe}$ to Co based multilayers, we find that it is $15 \%$ higher in the case of Fe. This is associated with an $8 \%$ lower packing fraction in the bcc structure of Fe relative to the fcc structure of Co. Thus it seems that there may be a correlation between the density of structure and the induced moment. This may be due to the less densely packed bct structure having narrower bands which tend to localize the magnetic electrons, making it a less itinerant system. In fact, bct Rh, when in proximity to $\mathrm{Fe}$, appears to sustain a moment even for those atoms not directly at the interface. ${ }^{7,8}$

\section{CLOSING REMARKS}

We have presented XMCD measurements of Co and $\mathrm{Rh}$ moments in a number of sputtered $\mathrm{Co} / \mathrm{Rh}$ (001) multilayer films. Here in the fct $\mathrm{Co} / \mathrm{Rh}$ system, we find that the $\mathrm{Rh}$ moment decreases in such a way as to indicate only interfacial polarization. This is quite different from what is observed for bct $\mathrm{Fe} / \mathrm{Rh}$ (001) multilayers, where $\mathrm{Rh}$ is in a metastable bct phase. Furthermore, bct $\mathrm{Rh}$ in $\mathrm{Fe} / \mathrm{Rh}$ multilayers possesses a larger moment than fct $\mathrm{Rh}$ in $\mathrm{Co} / \mathrm{Rh}$ multilayers. Thus the local crystalline as well as magnetic environment probably drives the observed $\mathrm{Rh}$ magnetic behavior.

\section{ACKNOWLEDGMENTS}

This work was supported by National Science Foundation CAREER Grant No. DMR-9623246. The Synchrotron Radiation Center is supported by the NSF under Grant No. DMR-9212658.
${ }^{1}$ S. S. P. Parkin, Phys. Rev. Lett. 67, 3598 (1991).

${ }^{2}$ A. Rampe, D. Hartmann, W. Weber, S. Popovic, M. Reese, and G. Günderodt, Phys. Rev. B 51, 3230 (1995).

${ }^{3}$ T. Kachel, W. Gudat, C. Carbone, E. Vescovo, S. Blügel, U. Alkemper, and W. Eberhardt, Phys. Rev. B 46, 12888 (1992).

${ }^{4}$ S. Zoll, A. Dinia, M. Gester, D. Stoeffler, H. A. M. van den Berg, A. Herr, R. Poinsot, and H. Rakoto, J. Magn. Magn. Mater. 165, 442 (1997).

${ }^{5}$ D. C. A. Stoeffler, J. Magn. Magn. Mater. 165, 62 (1997).

${ }^{6}$ H. Itoh, J. Inoue, and S. Maekawa, Phys. Rev. B 47, 5809 (1993).

${ }^{7}$ M. A. Tomaz, D. C. Ingram, G. R. Harp, E. Mayo, D. Lederman, and W. L. O'Brien, Phys. Rev. B 56, 5474 (1997).

${ }^{8}$ M. A. Tomaz, G. R. Harp, R. Wu, E. Mayo, D. Lederman, and W. L. O'Brien, J. Vac. Sci. Technol. A 16, 1336 (1998).

${ }^{9}$ J. L. Erskine and E. A. Stern, Phys. Rev. B 12, 5016 (1975).

${ }^{10}$ G. Schütz, W. Wagner, W. Wilhelm, P. Keinle, R. Zeller, R. Frahm, and G. Materlik, Phys. Rev. Lett. 58, 737 (1987).

${ }^{11}$ G. R. Harp, S. S. P. Parkin, W. L. O'Brien, and B. P. Tonner, Phys. Rev. B 51, 3293 (1995).
${ }^{12}$ M. A. Tomaz, G. R. Harp, E. Hallin, T. K. Sham, and W. L. O'Brien, J. Vac. Sci. Technol. A 16, 1359 (1998).

${ }^{13}$ B. M. Lairson, M. R. Visokay, R. Sinclair, S. Hagstrom, and B. M. Clemens, Appl. Phys. Lett. 61, 1390 (1992).

${ }^{14}$ L. Z. Mezey and J. Giber, Appl. Phys. A: Solids Surf. 35, 1569 (1984).

${ }^{15}$ The proportionality between the XMCD signal and the magnetic moment is only approximate. The error introduced into the magnetic moment measurement through the application of XMCD is about 10-20\% (Ref. 16) of the measured moment in the case of Co, and perhaps $30 \%$ for Rh.

${ }^{16}$ M. A. Tomaz, W. J. Antel, W. L. O’Brien, and G. R. Harp, Phys. Rev. B 55, 3716 (1997).

${ }^{17}$ M. A. Tomaz, W. J. Antel, W. L. O'Brien, and G. R. Harp, J. Phys.: Condens. Matter 9, L179 (1997).

${ }^{18}$ T. Lin, M. A. Tomaz, M. M. Shwickert, W. L. O'Brien, and G. R. Harp, Phys. Rev. B 58, 862 (1998).

${ }^{19}$ P. Carra, B. T. Thole, M. Altarelli, and X. Wang, Phys. Rev. Lett. 70, 694 (1993). 
${ }^{20}$ R. Wu and A. J. Freeman, Phys. Rev. Lett. 73, 1994 (1994).

${ }^{21}$ See $3 d, 4 d$, and $5 d$ Elements, Alloys, and Compounds, edited by H. P. J. Wijn, Landolt-Börnstein, New Series, Group 3, Vol. 19, Pt. A (Springer-Verlag, Berlin, 1986) and references therein.

${ }^{22}$ C. T. Chen, Y. U. Idzerda, H. J. Lin, N. V. Smith, G. Meigs, E.
Chaban, G. H. Ho, E. Pellegrin, and F. Sette, Phys. Rev. Lett. 75, 152 (1995).

${ }^{23}$ D. Weller, Y. Wu, J. Stöhr, M. G. Samant, B. D. Hermsmeier, and C. Chappert, Phys. Rev. B 49, 12888 (1994).

${ }^{24}$ V. L. Moruzzi and P. M. Marcus, Phys. Rev. B 39, 471 (1989). 